\section{Abordaje multiprofesional del paciente con úlcera de pie diabético y presencia de osteomielitis. Casos clínicos}

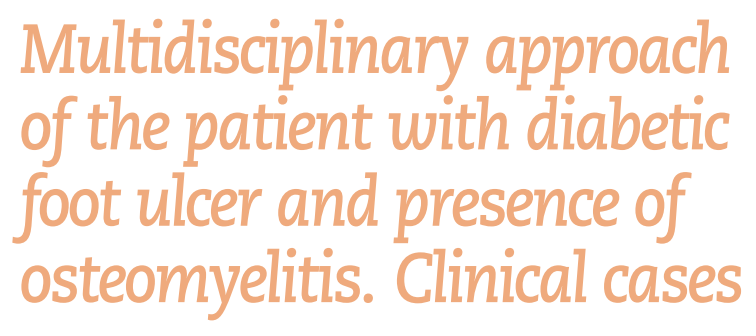

Juan Guillermo Ais Conde ${ }^{1}$ Mercedes Arranz Valentín ${ }^{2}$ Sandra Barrio Anaya ${ }^{3}$ Omar Alfonso Guerra Álvarez ${ }^{4}$ Isabel Mota San José ${ }^{2}$ Belén Pascual de la Fuente ${ }^{6}$ Juana Rituerto Cuerdo ${ }^{7}$ David Alcaide Carrillo,**

1. Cirujano. Unidad Multiprofesional de Heridas. Servicio de Cirugía. Hospital General de Segovia. Segovia. España.

2. Enfermera de Atención Primaria. Segovia. España.

3. Cirujana. Especialista en pie diabético. Unidad Multiprofesional de Heridas. Servicio de Cirugía. Hospital General de Segovia. Segovia. España.

4. Cirujano. Unidad Multiprofesional de Heridas. Servicio de Traumatología. Hospital General de Segovia. Segovia. España.

5. Especialista en pie diabético. Unidad Multiprofesional de Heridas. Supervisora del Servicio de Traumatología. Hospital General de Segovia. Segovia. España.

6. Enfermera. Especialista en pie diabético. Unidad Multiprofesional de Heridas. Hospital General de Segovia. Segovia. España.

7. Unidad Multiprofesional de Heridas. Supervisora del Servicio de Traumatología. Hospital General de Segovia. Segovia. España.

8. Enfermero de Atención Primaria. Especialista en pie diabético. Unidad Multiprofesional de Heridas. Segovia. España.

${ }^{*}$ Autor para correspondencia.

Correo electrónico: David.alcaidedue@gmail.com (David Alcaide Carrillo).

Recibido el 11 de abril de 2019; aceptado el 20 de mayo de 2019

\section{RESUMEN}

La osteomielitis del pie diabético puede presentar al clínico grandes desafíos diagnósticos y terapéuticos. La infección en pacientes con úlceras de pie diabético ocasiona una gran morbimortalidad. Las tasas de mortalidad, sobre todo cardiovascular, tras una úlcera y la amputación son muy elevadas, casi un 60\% de las personas fallecen en el transcurso de 5 años tras una amputación y casi un $40 \%$ en el caso de las úlceras. El objetivo del artículo es describir el procedimiento con el que se aborda la osteomielitis en pacientes con úlceras de pie diabético a través de un equipo multiprofesional. Metodología: A través de dos casos clínicos, se expone el planteamiento quirúrgico y médico para el abordaje de la osteomielitis. Resultados: La resolución tanto de la osteomielitis como de la cicatrización de la úlcera se apoya tanto en la especialización de los profesionales a nivel clínico como en garantizar la continuidad asistencial en los niveles de atención existentes, evitando la variabilidad y garantizando la prestación de los recursos necesarios de manera eficiente. Conclusiones: El

abordaje quirúrgico o médico de la osteomielitis precisa de un equipo cualificado que pueda plantear ambas estrategias de atención, consiguiendo la resolución del caso.

PALABRAS CLAVE: Pie diabético, osteomielitis, enfermería, continuidad de cuidados y úlcera de pie.

\section{ABSTRACT}

Osteomyelitis of the diabetic foot can present the clinician with great diagnostic and therapeutic challenges. Infection in patients with diabetic foot ulcers causes great morbidity and mortality. Mortality rates, especially cardiovascular, after an ulcer and amputation are very high, almost $60 \%$ of people die within 5 years after amputation and almost $40 \%$ in the case of ulcers. The objective of the article is to describe the procedure with which osteomyelitis is approached in patients with diabetic foot ulcers through a multiprofessional team. Methodology: Through two clinical cases, the surgical and medical approach for the osteomyelitis approach is exposed. Results: The resolution of both osteomyelitis and ulcer healing relies both on the specialization of professionals at a clinical level, and on ensuring continuity of care at existing levels of care, avoiding variability and guaranteeing the provision of Necessary resources efficiently. Conclusions: The surgical or medical approach of osteomyelitis requires a qualified team that can propose both strategies of care, obtaining the resolution of the case.

KEYWORDS: Diabetic foot, Osteomyelitis, Nurse, Continuity of care and foot ulcer. 


\section{INTRODUCCIÓN}

La osteomielitis del pie diabético puede presentar al clínico grandes desafíos diagnósticos y terapéuticos ${ }^{1}$. La osteomielitis se encuentra en torno al 50-60\% de los pacientes hospitalizados por una infección de pie diabético y aproximadamente entre el $10 \%$ y el $20 \%$ de las infecciones aparentemente menos graves que se presentan en el entorno ambulatorio ${ }^{2}$.

La osteomielitis de pie diabético representa actualmente un desafío tanto en el aspecto diagnóstico como terapéutico y muchas de las consecuencias de su padecimiento se relacionan claramente con un diagnóstico tardío, una derivación retrasada o un tratamiento mal indicado ${ }^{3}$.

Existen diferentes artículos en la literatura científica sobre el tratamiento de la osteomielitis, pero en la actualidad no se ha llegado a un consenso sobre cuál es la mejor opción terapéutica para resolverla ${ }^{3}$. En el presente artículo se describe el abordaje de la osteomielitis de 2 pacientes con úlceras de pie diabético (UPD) con presencia de osteomielitis, tratados hasta la resolución completa tanto de la infección como la cicatrización de la úlcera.

\section{Y OBSERVACIÓN Y PLAN DE DESARROLLO}

El abordaje de los casos clínicos presentados a continuación se lleva a cabo a través de un equipo multiprofesional en el desarrollo de la actividad de la Unidad Multiprofesional de Heridas (UMH) de la Gerencia de Asistencia Sanitaria de Segovia.

La estrategia para la continuidad de cuidados, proceso clave, permite disminuir la variabilidad y utilizar los recursos de manera eficiente.

El protocolo dirigido al pie diabético se estructura de la siguiente manera: valoración integral del paciente, incluyendo test y escalas propias y recomendadas por el panel de expertos: Escala TEXAS ${ }^{4}$, clasificación de la Sociedad Americana de Enfermedades Infecciosas (IDSA-PEDIS), clasificación del Grupo de Trabajo Internacional de Pie Diabético (IWGDF) ${ }^{5,6}$ y la clasificación RESVECH $2.0^{7}$ para el seguimiento de la úlcera:

- La estrategia se planifica entre los profesionales que componen la $\mathrm{UMH}$, incluidas unidades de consulta como el Servicio de Endocrinología y la Comisión de Infecciosas, que establece pauta de antibiótico, tras cultivo específico.
- El abordaje de la osteomielitis se lleva a cabo según las recomendaciones presentes en la tabla 1.

- Para la toma de cultivo se sigue el siguiente orden, según las posibilidades durante la realización: cultivo óseo, cultivo tisular, cultivo por aspiración y finalmente cultivo por hisopo.

- El protocolo de abordaje de la UPD por parte de la UMH consiste en descartar la isquemia (ecografía Doppler), descartar la infección (signos clínicos y prueba ósea más radiografía realizada al inicio y a las 2 semanas de evolución), descarga de la zona afectada (usando fieltro, zapato posquirúrgico o productos ortopédicos), desbridamiento del tejido desvitalizado e hiperqueratosis y cura en ambiente húmedo a través de los productos incluidos en el catálogo de la Gerencia de Asistencia Sanitaria de Segovia si no existe afectación arterial.

- Los apósitos principalmente empleados se describen en los casos clínicos; se fijan al lugar mediante un vendaje de sujeción o de contención con baja elasticidad si existe edema.

- El protocolo de la UMH de limpieza de la lesión incluye fomentos con Prontosan solución a través gasas introducidas en la cavidad, salvo contraindicación.

- El abordaje de la lesión se consensúa y coordina entre los diferentes niveles asistenciales.

- Para la exposición de los casos se solicitó el consentimiento informado firmado por los pacientes, informándoles de la divulgación a través de actividades científicas.

\section{У CASO CLÍNICO 1}

\section{Historia clínica}

Paciente de 57 años con antecedentes de diabetes mellitus tipo 2 y dislipemia. Presenta UPD de 6 meses de evolución a nivel de la articulación metatarsofalángica del $4 .^{\circ}$ dedo.

\section{Valoración}

General: consciente, orientado, hidratado y perfundido. UPD con área de celulitis que se extiende por el borde lateral y el dorso del antepié izquierdo. Presenta exudado purulento de la lesión (fig. 1 A y B).

Clasificación: TEXAS 3B, IDSA-PEDIS 2, REVECH 2.023.

Prueba ósea y radiografía: compatible con osteomielitis.

Exploración arterial: se explora el árbol arterial del miembro in-

Tabla 1. Indicaciones de tratamiento primario dependiendo de las características del paciente y de la lesión asociada a osteomielitis de pie diabético

- Úlceras confinadas en el antepié

- Pacientes con riesgo quirúrgico elevado (médicamente inestables)

- Pacientes bien vascularizados

- Ausencia de necrosis en tejidos blandos

- Úlceras fáciles de descargar

- Hueso y articulación no visible a través de la úlcera

- No disponibilidad de cirujano experto en el tratamiento de estas infecciones

- El paciente rechaza de forma rotunda la opción quirúrgica

- No son necesarios otros procedimientos quirúrgicos añadidos
- Hueso visualizable en el fondo de la úlcera. Presencia de necrosis en tejidos blandos

- Necesidad de drenaje de abscesos o colecciones. Fracaso del tratamiento con antibioticoterapia (90 días)

- Exposición articular (especialmente 1. ${ }^{\text {a }}$ metatarsofalángica). Necesidad de cirugía de descarga

- Destrucción ósea avanzada

- Pacientes en alto riesgo de toxicidad a antibioticoterapia (especialmente en pacientes nefrópatas). Infecciones causadas por patógenos resistentes a antibioticoterapia disponibles 

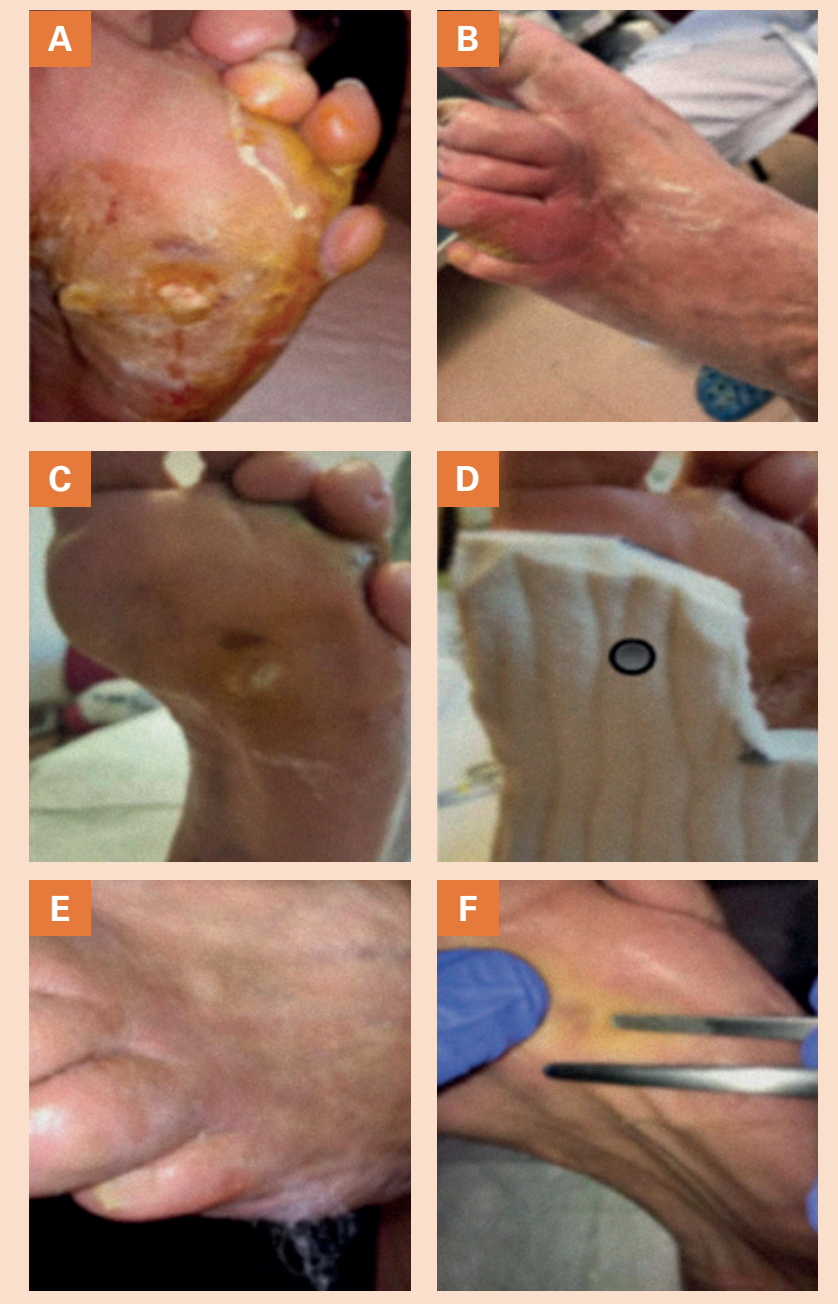

Figura 1. A) 03-05-2018: primera visita. Planta del pie. B) 03-05-2018: primera visita, dorso del pie. C) Imagen visita 28-05-2018: tras retirada. Eliminación de la hiperqueratosis. D) 28-05-2018.. descarga con fieltro. E) 14-06-2018: resolución de la infección. F) 23-07-2018: cicatrización completa de la lesión.

ferior izquierdo desde femoral común distal a tibiales anterior y posterior distales. Los flujos son trifásicos y de amplitud normal a todos los niveles.

\section{Diagnóstico}

UPD neuropática.

\section{Plan de actuación y tratamiento}

El plan de actuación esta descrito en el apartado protocolo de asistencia dirigido al pie diabético (fig. $1 \mathrm{C} \mathrm{y} \mathrm{D).} \mathrm{Se} \mathrm{realizan} \mathrm{tres} \mathrm{curas} \mathrm{semanales}$ (lunes, miércoles y viernes) programadas. Los productos utilizados fueron, inicialmente, Biatain Alginate $\mathrm{Ag}^{\oplus}$ para el control de la infección local y gestión adecuada del abundante exudado, y posteriormente se empleó Urgo Start ${ }^{\circledR}$ cuando el exudado pasó a ser moderado-leve hasta la epitelización. Se utilizó fieltro de descarga $(15 \mathrm{~mm})$ y zapato posquirúrgico de tacón trasero.
Para el abordaje médico de la osteomielitis (tabla 1), se utilizó levofloxacino por vía intravenosa, $500 \mathrm{mg} / 100 \mathrm{ml}$ durante 6 semanas, acudiendo el paciente diariamente al hospital, ya que rehusó ingreso hospitalario.

\section{Evolución}

La lesión cicatrizó por completo a los 2 meses y 20 días (03/05/2018 a 23/07/2018). Actualmente, el paciente está incluido en el programa de seguimiento de pacientes con alto riesgo de pie diabético sin presentar úlcera (fig. $1 \mathrm{E}$ y F).

\section{У CASO CLÍNICO 2}

\section{Historia clínica}

Paciente de 68 años con antecedentes de diabetes mellitus tipo 2, insuficiencia renal aguda, insuficiencia venosa crónica, bypass aortocoronario, hipertensión arterial y dislipemia. Paciente intervenido el día 21/06/2018 por presentar fractura-luxación de Lisfranc inveterada, realizándose reducción y osteosíntesis con tornillo tipo Acutrac ${ }^{\bullet}$ desde cuña medial a base de primer metatarsiano.

Ingresa nuevamente el 29/10/19 en hospitalización en el Servicio de Cirugía por dehiscencia de sutura.

\section{Valoración}

UPD en mediopié en paciente con neuroartropatía de Charcot. Presenta exposición ósea, herida con abundante exudado y tejido de hipergranulación.

Clasificación: TEXAS 3B, IDSA-PEDIS 3, REVECH 2.0 26.

\section{Diagnóstico}

UPD neuropática en neuroartropatía de Charcot.

\section{Plan de actuación y tratamiento}

Es atendido por la UMH el 13/11/18, iniciando terapia de presión negativa y antibioticoterapia intravenosa sin que exista evolución. El 20/11/2018 (fig. 2 A) es atendido por el traumatólogo de referencia para la UMH. En dicha fecha se realiza limpieza, desbridamiento, extracción de material de osteosíntesis con exéresis completa de cuña interna y hueso periarticular afectado por osteomielitis, el cual es enviado a analizar.

El cultivo es positivo para Klebsiella pneumoniae, por lo que se inicia tratamiento de antibioticoterapia intravenosa con meropenem 1 gramo.

El plan de actuación está descrito en el apartado proceso asistencial dirigido al pie diabético. Se realizó descarga con bota ortoprotésica inmovilizadora (bota Walker ${ }^{\circledR}$ ) inicialmente con cura diaria hasta control del exudado, pasando a 3 curas semanales (lunes, miércoles y viernes) programadas. Como apósito primario se usó Biatain Alginate Ag ${ }^{\oplus}$, IodoSorb ${ }^{\oplus}$ para el control de la infección local, y como secundario se emplea Biatain Alginate ${ }^{\circledast}$. Cuando el exudado pasó a ser moderado-leve utilizamos Urgo Start ${ }^{\circledast}$ hasta la epitelización.

Tras el abordaje quirúrgico de la osteomielitis (tabla 1) se estableció tratamiento con Septrin forte ${ }^{\otimes} 800 \mathrm{mg} / 160 \mathrm{mg}$ desde el 11/12/18 al 11/01/2019 durante su hospitalización (fig. 2 E).

Exploración arterial: se explora el árbol arterial del miembro inferior izquierdo desde femoral común distal a tibiales anterior y posterior distales. Se aprecian pequeńas placas de ateroma calcificadas sin detectarse estenosis significativas. Los flujos son trifásicos y de amplitud normal a todos los niveles. 

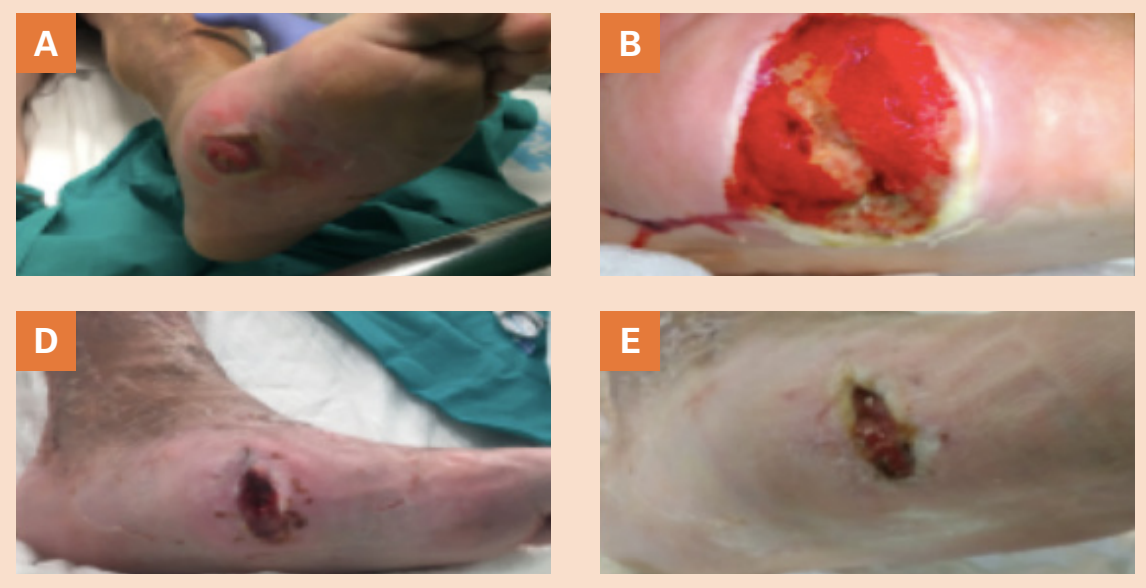
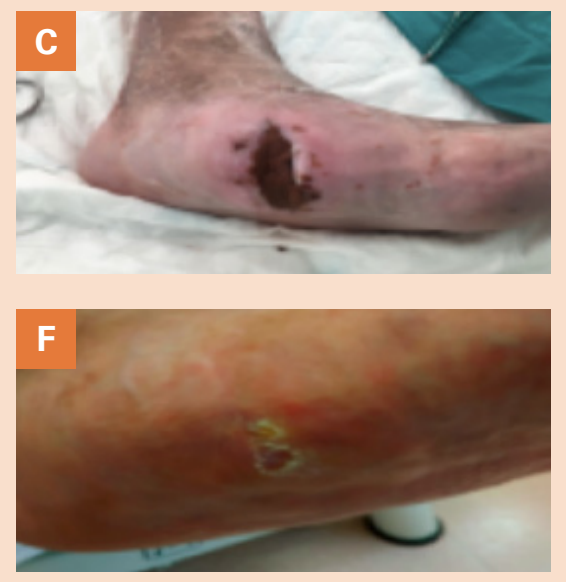

Figura 2. A) 13-11-2018: antes de la intervención quirúrgica. B) 22-11-2018: primera visita a la UMH tras la intervención. C) 19-12-2018: cura con lodosorb . D) 21-12-2018: presencia de tejido de granulación en el lecho. E) 11/01/19: evolución. F) 25/01/2019: resolución de la herida.

\section{Evolución}

La lesión cicatrizó completamente a los 2 meses y 5 días (25/01/19). Actualmente, el paciente está incluido en el programa de seguimiento de pacientes con alto riesgo de pie diabético sin presentar úlcera (fig. $2 \mathrm{~F}$ ).

\section{Discusión}

El abordaje planteado por la UMH a través de profesionales especializados en pie diabético y úlceras crónicas en coordinación con las unidades de consulta y la enfermera de atención primaria garantizando la continuidad asistencial fue eficaz, consiguiendo abordar la osteomielitis y el cierre completo de la lesión. Estrategias como la elaborada por la Dirección General de Asistencia Sanitaria del Gobierno de Aragón hacen referencia a cómo las UPD puede ocasionar importante discapacidad, y en ausencia de control del paciente y manejo inadecuado puede derivar hacia un resultado desfavorable con una posible amputación del pie o la pierna ${ }^{8}$. Las tasas de mortalidad, sobre todo cardiovascular, tras una úlcera y la amputación son muy elevadas; casi un $60 \%$ de las personas fallecen en el transcurso de 5 años tras una amputación y casi un $40 \%$ en el caso de las úlceras ${ }^{9-12}$.

La osteomielitis de pie diabético representa actualmente un desafío tanto en el aspecto diagnóstico como terapéutico, y muchas de las consecuencias de su padecimiento se relacionan claramente con un diagnós- tico tardío, una derivación retrasada o un tratamiento mal indicado ${ }^{3}$. Los principales problemas en el diagnóstico de osteomielitis son que hay un retraso en la capacidad para detectar cambios óseos en la infección temprana en las radiografías simples, mientras que, más tarde, cuando se producen cambios óseos, puede ser difícil distinguir en estudios de imagen aquellos causados por la infección de aquellos relacionados con la neuroartropatía de Charcot ${ }^{2}$. El seguimiento y cuidado especializados del paciente a través de un abordaje integral, haciendo uso de test y escalas de referencia, pruebas como la prueba ósea conjunta con radiografía, seguimiento de marcadores sanguíneos específicos como la velocidad de sedimentación globular y la proteína $\mathrm{C}$ reactiva, así como aspectos clínicos de la evolución de la herida, son componentes clave para el seguimiento y abordaje de este problema y su resolución

\section{Agradecimientos}

Nuestro agradecimiento a todo el equipo que compone la Unidad Multiprofesional de Heridas, destacando a los 3 enfermeros expertos en heridas crónicas y pie diabético que no aparecen en la autoría, así como a los profesionales con los que habitualmente colaboramos en nuestra actividad.

\section{Conflictos de intereses}

Los autores manifiestan no tener conflicto de intereses con ningún producto de los referidos, ya que pertenecen al catálogo de productos sanitarios disponible en dicha gerencia.

\section{У BIBLIOGRAFÍA}

1. Lipsky BA. Bone of contention: diagnosing diabetic foot osteomvelitis. Clin Infect Dis. 2008:47:528-30

2. Bakker K, Apelqvist J, Lipsky BA, Van Netten JJ, Schaper NC. The 2015 IWGDF guidance documents on prevention and management of foot problems in diabetes: Development of an evidence-based global consensus. Diabetes Metab Res Rev. 2016;32:2-6.

3. Lázaro-Martínez JL, Tardáguila-García A, García Klepzig JL. Actualización del diagnóstico y tratamiento del pie diabético con osteomielitis. Endocrinol Diabetes Nutr. 2017:64(2):100-8.

4. Lavery LA, Armstrong DG, Harkless LB. Classification of Diabetic Foot Wounds. J Foot Ankle Surg. 1996;35:528-31.

5. Schaper NC. Diabetic foot ulcer classification system for research purposes: a progress report on criteria for including patients in re- search studies. Diabetes Metab Res Rev. 2004;20(suppl 1):90-5.

6. Lipsky BA, Berendt AR, Cornia PB, Pile JC, Peters EJG, Armstrong DG, et al. 2012 Infectious Diseases Society of America clinical practice guideline for the diagnosis and treatment of diabetic foot infections. Clin Infect Dis. 2012:54:e132-73.

7. Ibars-Moncasi, P, San Sebastián-Domínguez JA, Soldevilla-Agreda J. Conjunto Mínimo Básico de Datos en registros de Úlceras por Presión (CMBD-UPP). Serie Documentos de Posicionamiento GNEAUPP no 11.2012.

8. Aragon N, Cardiel MB, Borobio GM, Carlos J, Bravo A, Gil SB. Atención al pie diabético en Aragón. [Internet]. 2018. Disponible en: https:// www.aragon.es/documents/20127/674325/Atencion pie diabetico Aragon_mayo_2018.pdf/6d8325a3-270d-c252-88cd-d9606325f26c
9. Kerr M, Rayman G Jeffcoate WJ. Cost of diabetic foot disease to the National Health Service in England. Diabet Med. 2014;31(12):1498-504.

10. Jupiter DC, Thorud JC, Buckley CJ, Shibuya N. The impact of foot ulceration and amputation on mortality in diabetic patients. I: From ulceration to death, a systematic review. Int Wound J 2016;13(5):892-903

11. Zhang P, Lu J, Jing Y, Tang S, Zhu D, Bi Y. Global epidemiology of diabetic foot ulceration: a systematic review and meta-analysis. Ann Med. 2016;1:1-21.

12. Internal Clinical Guidelines team. Diabetic Foot Problems: Prevention and Management. London: National Institute for Health and Care Excellence (UK); 2015 Aug. PMID: 26741017. 